Article

\title{
Genome-Scale Metabolic Modeling Reveals Metabolic Alterations of Multidrug-Resistant Acinetobacter baumannii in a Murine Bloodstream Infection Model
}

\author{
Jinxin Zhao ${ }^{1}\left(\right.$, Yan Zhu ${ }^{1} * \mathbb{\infty}$, Jiru Han ${ }^{2}$, Yu-Wei Lin ${ }^{1}$, Michael Aichem ${ }^{3}$, Jiping Wang ${ }^{1}$, \\ Ke Chen ${ }^{1}$, Tony Velkov ${ }^{4}{ }^{\oplus}$, Falk Schreiber ${ }^{3}$ and Jian $\mathrm{Li}^{1, *}$ \\ 1 Infection and Immunity Program, Department of Microbiology, Biomedicine Discovery Institute, \\ Monash University, Clayton, VIC 3800, Australia; jinxin.zhao@monash.edu (J.Z.); \\ Yu-Wei.Lin@monash.edu (Y.-W.L.); Jiping.Wang@monash.edu (J.W.); Ke.Chen@monash.edu (K.C.) \\ 2 Population Health and Immunity Division, The Walter and Eliza Hall Institute of Medical Research, \\ Parkville, VIC 3052, Australia; han.ji@wehi.edu.au \\ 3 Department of Computer and Information Science, University of Konstanz, 78457 Konstanz, Germany; \\ michael.aichem@uni-konstanz.de (M.A.); falk.schreiber@uni-konstanz.de (F.S.) \\ 4 Department of Pharmacology and Therapeutics, University of Melbourne, Melbourne, VIC 3010, Australia; \\ tony.velkov@unimelb.edu.au \\ * Correspondence: yan.zhu@monash.edu (Y.Z.); jian.li@monash.edu (J.L.); \\ Tel.: +61-3-99029178 (Y.Z.); +61-3-99039172 (J.L.); Fax: +61-3-99056450 (J.L.)
}

Received: 19 October 2020; Accepted: 13 November 2020; Published: 16 November 2020

check for updates

\begin{abstract}
Multidrug-resistant (MDR) Acinetobacter baumannii is a critical threat to human health globally. We constructed a genome-scale metabolic model $i \mathrm{AB} 5075$ for the hypervirulent, MDR A. baumannii strain AB5075. Predictions of nutrient utilization and gene essentiality were validated using Biolog assay and a transposon mutant library. In vivo transcriptomics data were integrated with $i \mathrm{AB} 5075$ to elucidate bacterial metabolic responses to the host environment. iAB5075 contains 1530 metabolites, 2229 reactions, and 1015 genes, and demonstrated high accuracies in predicting nutrient utilization and gene essentiality. At $4 \mathrm{~h}$ post-infection, a total of 146 metabolic fluxes were increased and 52 were decreased compared to $2 \mathrm{~h}$ post-infection; these included enhanced fluxes through peptidoglycan and lipopolysaccharide biosynthesis, tricarboxylic cycle, gluconeogenesis, nucleotide and fatty acid biosynthesis, and altered fluxes in amino acid metabolism. These flux changes indicate that the induced central metabolism, energy production, and cell membrane biogenesis played key roles in establishing and enhancing A. baumannii bloodstream infection. This study is the first to employ genome-scale metabolic modeling to investigate $A$. baumannii infection in vivo. Our findings provide important mechanistic insights into the adaption of A. baumannii to the host environment and thus will contribute to the development of new therapeutic agents against this problematic pathogen.
\end{abstract}

Keywords: Acinetobacter baumannii; genome-scale metabolic modeling; transcriptomics; bacteremia; RNA-seq

\section{Introduction}

Multidrug-resistant (MDR) Acinetobacter baumannii has become a critical threat to human health globally [1]. It has a high incidence of nosocomial infections, including bacteremia, pneumonia, wound sepsis, and urinary tract infections [2]. Recently, the World Health Organization (WHO) identified 
carbapenem-resistant $A$. baumannii as one of three top-priority pathogens that urgently require novel antimicrobial therapeutics [3].

Antibiotics target essential components of bacterial growth, such as DNA replication, translation, and peptidoglycan biosynthesis [4]. However, resistance to antibiotics can develop rapidly in bacteria [5]. Virulence factors, such as outer membrane proteins, secretion systems, phospholipases, and iron acquisition systems, promote bacterial intracellular replication, cell adhesion, and invasion, and are crucial for pathogens to adapt to the host environment [6]. Strategies to inhibit virulence factors represent a promising alternative therapeutic option for the treatment of severe infections caused by MDR bacteria [7]. Dual RNA-seq is increasingly used to identify the key factors contributing to host adaptation during infection [8-11]. Several key metabolic pathways (e.g., the phenylacetic acid metabolism pathway) are associated with the establishment of $A$. baumannii infection in vivo [12,13]; however, it remains unclear how exactly host adaption is controlled by the complex bacterial metabolic network during infection.

Genome-scale metabolic modeling (GSMM) is increasingly used to decipher the metabolic changes in pathogens under infection or antibiotic treatment conditions [14-16]. Integration with multi-omics data enabled GSMMs to accurately describe cellular metabolism [17]. In the present study, we report the development and validation of the first GSMM for A. baumannii MDR strain AB5075. By incorporating in vivo transcriptomics data as constraints, our model was able to identify the significant metabolic changes of AB5075 during infection in mice. This is the first integrative modeling of A. baumannii infection in vivo and provides key mechanistic information regarding bacterial metabolic changes in response to the host immune system. Such information will facilitate optimization of antibiotic therapy for infections caused by A. baumannii.

\section{Materials and Methods}

\subsection{Bacterial Strain and Growth Condition}

Hypervirulent MDR A. baumannii AB5075 was obtained from the University of Washington and stored at $-80^{\circ} \mathrm{C}$ in tryptone soy broth (TSB, Oxoid Australia) with $20 \%$ glycerol. Prior to experiments, AB5075 was sub-cultured onto nutrient agar and incubated at $37^{\circ} \mathrm{C}$ overnight. A single colony was then selected and grown overnight in $20 \mathrm{~mL}$ of cation-adjusted Mueller-Hinton broth (MHB; Oxoid, Australia; $20-25 \mathrm{mg} \cdot \mathrm{L}^{-1} \mathrm{Ca}^{2+}$ and $10-12.5 \mathrm{mg} \cdot \mathrm{L}^{-1} \mathrm{Mg}^{2+}$ ), from which a 1:100 dilution was performed in fresh broth to prepare mid-logarithmic cultures $\left(\mathrm{OD}_{600 \mathrm{~nm}}=0.4\right.$ to 0.6$)$. All broth cultures were incubated at $37^{\circ} \mathrm{C}$ in an open-air shaker (200 rpm). Final bacterial suspensions were concentrated to $1 \times 10^{10} \mathrm{CFU} \cdot \mathrm{mL}^{-1}$ in sterile saline.

\subsection{Animals}

Animal experiments were approved by Monash University Animal Ethics Committee. For all animal experiments, Swiss mice (female, 8-10 weeks, body weight 25-35 g) were obtained from Monash Animal Services. Animals were handled, fed, and housed according to the criteria of the Australian Code of Practice for the Care and Use of Animals for Scientific Purposes [18]. Food and water were available ad libitum.

\subsection{Non-Neutropenic Murine Bacteremia Model}

A non-neutropenic murine bacteremia infection model was employed in this study. Mice were briefly anesthetized via placement into an isoflurane induction chamber. Anesthetized mice were placed on a Perspex support in a vertical upright position, which allowed the mice to be temporarily immobilized. Two independent groups of mice ( $n=3$ per group) were injected with $10 \mu \mathrm{L}$ of bacterial suspension (approximately $1.0 \times 10^{9}$ colony-forming units (CFUs) in early logarithmic phase) via an intravenous injection at $0 \mathrm{~h}$ and then placed onto a warm pad for rapid recovery. Our preliminary studies showed that bacterial infection established in mice after approximately $2 \mathrm{~h}$ post bacterial 
inoculation, and bacterial load peaked at approximately $4 \mathrm{~h}$ post bacterial inoculation. Therefore, we used 2 and $4 \mathrm{~h}$ post inoculation in this study to represent the establishment of bacterial infection and the maximum bacterial load, respectively. Samples were collected for RNA extraction and sequencing at Genewiz (paired-end 150 bp, Illumina HiSeq, Suzhou, China). Raw reads were submitted to the Sequence Read Archive Database (accession number: SRS7522398) [19].

\subsection{Construction of the Genome-Scale Metabolic Model (GSMM) iAB5075}

The genome annotation of A. baumannii AB5075 (i.e., AB5075-UW) was obtained from the PGAT database [20] and a draft model was initially constructed using CarveMe [21]. Further manual curation was conducted, including (i) adding transport reactions and extracellular metabolites; (ii) detecting and filling pathway gaps; and (iii) checking the mass and charge balance for each reaction. The obtained model was compiled in Systems Biology Markup Language (SBML) [22].

\subsection{Biolog Assay and Prediction of Nutrient Utilizations}

AB5075 was subcultured onto nutrient agar and incubated at $37^{\circ} \mathrm{C}$ for $20 \mathrm{~h}$. Biolog phenotype microarrays (PMs; Cell Biosciences, Australia) were employed to test the utilization of 190 carbon and 95 nitrogen sources, with 3 independent biological replicates. Bacterial growth was detected after 18 and $24 \mathrm{~h}$ of incubation at $37^{\circ} \mathrm{C}$ by measuring the optical density at $595 \mathrm{~nm}$ using an Infinite M200 microplate reader (Tecan, Mannedorf, Switzerland). Readings with $\geq 1$.5-fold of blank media controls were considered as utilization of nutrients. The constructed GSMM iAB5075 was then employed to predict the bacterial growth on a chemically defined media with 190 individual carbon sources and 95 nitrogen sources using flux balance analysis (FBA) method with COBRA toolbox 3.0 [23]. Biomass formation was optimized with the maximum specific carbon nutrient uptake rate set at $10 \mathrm{mmol} \cdot \mathrm{gDW}^{-1} \cdot \mathrm{h}^{-1}$ under aerobic condition [24]:

$$
\begin{gathered}
\max \quad v_{\text {biomass }}, \\
\text { s.t. } \mathbf{S} \cdot \mathbf{v}=0, \\
v_{j}^{\min } \leq v_{j} \leq v_{j}^{\max }, j=1,2, \cdots, n,
\end{gathered}
$$

where $\mathbf{S}$ represents the stoichiometric matrix with $m$ metabolites and $n$ reactions. Each flux $v_{j}$ is constrained by the lower bound $v_{j}^{\min }$ and upper bound $v_{j}^{\max }$. The prediction accuracy was calculated by comparison with Biolog experimental results as previously described [21]. Briefly, a true positive (TP) or true negative (TN) was considered as a correct prediction of a utilizable or non-utilizable nutrient source for growth, respectively; a false negative (FN) or false positive (FP) was considered an incorrect prediction of a utilizable or non-utilizable nutrient source, respectively. The prediction accuracy was then calculated by:

$$
\text { overall accuracy }=\frac{(T P+T N)}{(T P+T N+F P+F N)}
$$

The Matthews correlation coefficient (MCC) [25] was then calculated by:

$$
\frac{T P \times T N-P F \times P N}{\sqrt{(T P+F P)(T P+F N)(T N+F P)(T N+F N)}}
$$

\subsection{Gene Essentiality Analysis}

In silico single-gene deletion was conducted using both FBA and minimization of metabolic adjustment (MOMA) algorithms [14]. FBA predicts growth and metabolic fluxes based on the assumption that growth efficiency has evolved to an optimal point using linear programming. In contrast to FBA, MOMA does not assume optimality of growth. Instead, MOMA relaxes the 
assumption of optimal growth flux for gene deletions by performing distance minimization in flux space using quadratic programming [14]. Nutrient uptake constraints were set according to ingredients of chemically defined $\mathrm{M} 9, \mathrm{MH}$, and Luria-Bertani (LB) media. Essential metabolites were predicted by calculating the growth rate when switching off the corresponding consuming fluxes, and essential reactions were predicted by setting each reaction flux to zero while maximizing the biomass formation [15]. The recently generated three-allele transposon mutant library for A. baumannii AB5075 was employed as a reference to assess the prediction accuracy as previously described [26].

\subsection{Calculating Metabolic Fluxes with Transcriptomics Constraints}

The in vivo RNA-Seq data (accession number: SRS7522398) of AB5075 were incorporated to $i$ AB5075 using INIT (Integrative Network Inference for Tissues) algorithm, which is formulated as a mixed integer linear programming problem (MILP) shown below [27]:

$$
\begin{gathered}
\max \sum_{i \in R} w_{i} y_{i} \\
\text { s.t. } \quad \mathbf{S} \cdot \mathbf{v}=0, \\
\left|v_{i}\right| \leq 1000 y_{i}, \\
\left|v_{i}\right|+1000\left(1-y_{i}\right) \geq \varepsilon, \\
v_{i} \geq 0, i \in \text { irreversible rxns, } \\
y_{i} \in\{0,1\} .
\end{gathered}
$$

The parameter $\varepsilon$ is an arbitrarily small positive number, which was set by default in COBRA toolbox; $w_{i}$ is the weight of the $i$ th reaction calculated using transcriptomic data; and $y_{i}$ is an integer variable indicating either including $\left(y_{i}=1\right)$ or excluding $\left(y_{i}=0\right)$ of the $i$ th reaction in the extracted model [23]. The optimal trade-off between including and removing reactions was based on their weights $(w)$ [27], which were calculated as follows:

$$
w_{i, j}=5 \log \left(\frac{\text { RPKM }_{i, j}}{\text { Average }_{i}}\right)
$$

Specifically, RPKM (reads per kilobase per million) values were calculated using edgeR and employed to calculate the weights $w$ [28]. Briefly, all the RNA-seq data of AB5075 available from the public database (Gene Expression Omnibus) and our previous studies were collected and used for estimation of the average expression level of each gene. If the RPKM of the $i$ th gene in the $j$ th condition is higher than its average across all the samples, $w_{i, j}$ is positive. Otherwise, $w_{i, j}$ is negative. An weights-containing objective function is then maximized to achieve the best agreement of the calculated metabolic fluxes with the transcriptomic data. Moreover, to further improve the prediction, additional protein crowding constraints were incorporated into the extracted model. The total abundance of metabolic enzyme $(P)$ was set to $0.25 \mathrm{~g} \cdot \mathrm{gDW}^{-1}$ according to previous Escherichia coli data due to the limited quantitative proteomics data in A. baumannii [14]. The molecular weight $\left(M W_{k}\right)$ of $k$ th protein was calculated based on its amino acid sequence:

$$
\begin{gathered}
\max v_{\text {biomass }}=\mathbf{c} \cdot \mathbf{v}^{\mathrm{T}}, \\
\text { s.t. } \mathbf{S} \cdot \mathbf{v}=0 \\
v_{k}^{\min } \leqq v_{k} \leqq v_{k}^{\max }, \\
v_{k} \leqq k_{\text {cat }, k} \cdot e_{k}, \\
\sum e_{k} M W_{k}=P,
\end{gathered}
$$


where reaction flux $v_{k}$ is limited by the enzyme turnover rate $k_{\text {cat }, k}$ and enzyme molar abundance $e_{k}$ as previously described $[14,29]$. Due to the lack of enzyme kinetic data in $A$. baumannii, an averaged $k_{\text {cat }}$ $\left(65 \mathrm{~s}^{-1}\right)$ in E. coli was used unless specific $k_{\text {cat }}$ values were available in BRENDA database (e.g., $2740 \mathrm{~s}^{-1}$ for xanthine dehydrogenase catalyzing reaction R_HXAND [30], $1 \mathrm{~s}^{-1}$ for methionyl aminopeptidase catalyzing reaction R_AMPTALAGLN [31], $11.14 \mathrm{~s}^{-1}$ for D-Alanine-D-alanine ligase catalyzing reaction R_ALAALAr [32], $34 \mathrm{~s}^{-1}$ for NAD ${ }^{+}$synthase catalyzing reaction R_NADS1 [33]). FBA was conducted using COBRA toolbox 3.0 [23] in MATLAB environment and the significantly perturbed metabolic fluxes were identified using a Z-score based approach [34]. Briefly, genome-scale metabolic models for AB5075 at 2 and $4 \mathrm{~h}$ post-infection were obtained with the growth constraints $\left(0.21\right.$ and $0.30 \mathrm{~h}^{-1}$ for 2 and $4 \mathrm{~h}$ post-infection, respectively) calculated from the bacterial viable counts. Metabolic solution space was sampled with 10000 random points with the ll-ACHRB (loopless Artificially Centered Hit-and-Run on a Box) algorithm and linear programming solver Gurobi 9.0 [35]. Statistical significance of differential flux distributions was estimated using a Z-score method. Differential metabolic fluxes were filtered with FDR $<0.05$ and fold change $>2$.

\section{Results}

\subsection{Construction of the Genome-Scale Metabolic Model iAB5075}

A draft model involving 1480 metabolites and 2184 reactions was developed by CarveMe based on genome annotation. Further manual curation was conducted, including (i) addition of transport and exchange reactions to enable nutrient uptake and by-product secretion, and (ii) filling pathway gaps. Acinetobactin is the essential siderophore for iron uptake in A. baumannii [36]. To make our GSMM more representative of $A$. baumannii, 15 reactions and 20 metabolites involved in acinetobactin biosynthesis were added to the draft model. Altogether, a final model $i$ AB5075 was obtained that involved 1530 metabolites and 2229 reactions (Table 1). iAB5075 includes 1015 genes representing $26.1 \%$ of the genome and 18 of 23 clusters of orthologous groups (COG, Figure 1); the majority of genes were from amino acid transport and metabolism, energy production and conversion, and pathways involving transport and metabolism of inorganic ion, coenzyme, lipid, carbohydrate and nucleotide, as well as cell envelope biogenesis (Figure 1). iAB5075 contained intracellular, extracellular, and periplasmic compartments (Table 1). Compared with other GSMMs (i.e., iATCC19606v2 for strain ATCC 19606 and $i$ CN718 for strain AYE), iAB5075 represents the most comprehensive GSMM for A. baumannii determined thus far (Table 1).

Table 1. Genome contents and model components.

\begin{tabular}{ccccc}
\hline Content & iAB5075 draft $^{2}$ & iAB5075 & iATCC19606v2 & iCN718 \\
\hline Genome size $(\mathrm{Mb})$ & \multicolumn{2}{c}{3.97} & 3.98 & 3.90 \\
Assembly status & \multicolumn{2}{c}{ Complete } & Complete & Complete \\
GC content & \multicolumn{2}{c}{$39.1 \%$} & $39.2 \%$ & $39.0 \%$ \\
No. of genes & \multicolumn{2}{c}{3895} & 3805 & 3694 \\
No. of CDS & \multicolumn{2}{c}{3771} & 3663 & 3600 \\
No. of reactions & 2184 & 2229 & 2114 & 1016 \\
No. of metabolites & 1480 & 1530 & 1422 & 890 \\
No. of involved genes & 1010 & 1015 & 1009 & 718 \\
Compartment $^{\mathrm{a}}$ & $3(\mathrm{c}, \mathrm{p}, \mathrm{e})$ & $3(\mathrm{c}, \mathrm{p}, \mathrm{e})$ & $3(\mathrm{c}, \mathrm{p}, \mathrm{e})$ & $2(\mathrm{c}, \mathrm{e})$ \\
Prediction accuracy $^{\mathrm{b}}$ & $72.8 \% / 74.3 \%$ & $86.3 \% / 87.6 \%$ & $85.6 \% / 82.1 \%$ & $83.7 / 80 \%$ \\
MCC $^{\mathrm{b}}$ & $0.58 / 0.26$ & $0.76 / 0.28$ & $0.68 / 0.33$ & $0.72 / 0.32$ \\
\hline
\end{tabular}

Comparison of genome and GSMM features among isolates AB5075 (iAB5075), ATCC19606 (iATCC19606v2) and AYE (iCN718) [14-16]; ${ }^{a}$ the compartment in the models; c: intracellular compartment; p: periplasmic compartment; e: extracellular compartment; ${ }^{b}$ Prediction accuracy and Mathews correlation coefficients for Biolog and gene essentiality results. 


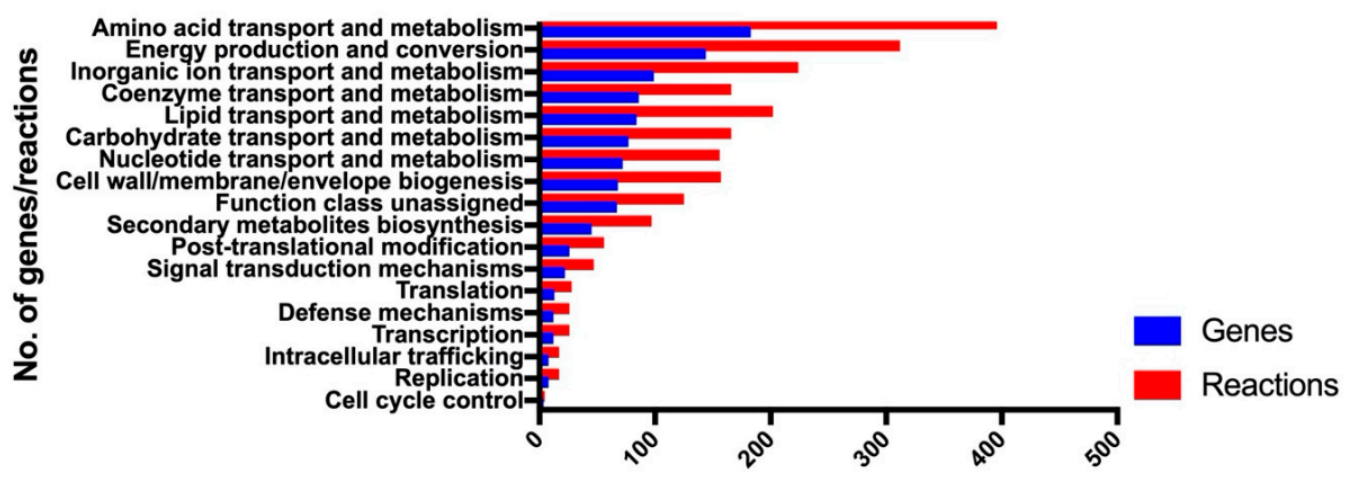

Figure 1. Clusters of orthologous (COG) functional classification of the genes involved in $i$ AB5075.

\subsection{Prediction of Bacterial Growth on Various Nutrients}

With additional protein crowding constraints, model $i$ AB5075 predicted bacterial exponential growth at 1.87, 2.64, 0.97, and $0.72 \mathrm{~h}^{-1}$ in Luria-Bertani (LB) media, Mueller-Hinton (MH) media, M9 media supplemented with citrate (M9C), and M9 media supplemented with succinate (M9S), respectively; these specific growth rates are consistent with experimental observations (Figure S1). Moreover, $i$ AB5075 predicted that strain AB5075 was able to utilize 41 of 190 carbon sources and 34 of 95 nitrogen sources (Figure 2). Manual curation was conducted to enhance the prediction accuracy. A false positive was usually caused by incorrectly involving transport reactions during automatic model construction; these reactions were then removed from the draft model according to the literature. While a false negative was likely caused by mis-annotation of gene functions; extensive gap filling with homology search was conducted to add the missing reactions. For example, predictions using the draft model showed that AB5075 was unable to grow with glycyl-L-asparagine or L-alanyl-L-histidine as the sole nitrogen sources; whereas Biolog results indicated that it could. We then added the related transport reactions (R_DIPEPabc8 and R_DIPEPabc5, respectively) and two aminopeptidase reactions (R_AMPTGLYASN and R_AMPTALAHIS, ABUW_2837 and ABUW_3646, with 57\% and 58\% identity of their E. coli homologs, respectively) to enable in silico utilization of these two nutrients. Further prediction under anaerobic conditions was undertaken using $i$ AB5075. The prediction results showed no growth under anaerobic conditions and it is consistent with the fact that A. baumannii is an obligate aerobe. The overall prediction accuracy of nutrient utilization achieved was high $(86.3 \%$; Fisher's exact test, $P=2.2 \times 10^{-16}$ ) compared with Biolog assay in which AB5075 grew on 59 carbon sources and 46 nitrogen sources (Figure 2). Core reactome analysis was conducted for all flux-carrying reactions in $\mathrm{LB}, \mathrm{MH}$, and $\mathrm{M} 9$ media supplemented with 73 different carbon and nitrogen sources. A core reactome containing 202 flux-carrying reactions was identified (Figure S2a), with 224 and 236 reactions as core reactomes for carbon and nitrogen sources, respectively. These core reactions are involved in 13 COG groups, including energy production and conversion, and transport and metabolism of lipid and amino acid (Figure S2b). Overall, the pan reactome consists of 1240 flux-carrying reactions, with 1139 and 1068 flux-carrying reactions specifically detected for growth on carbon and nitrogen sources, respectively. 


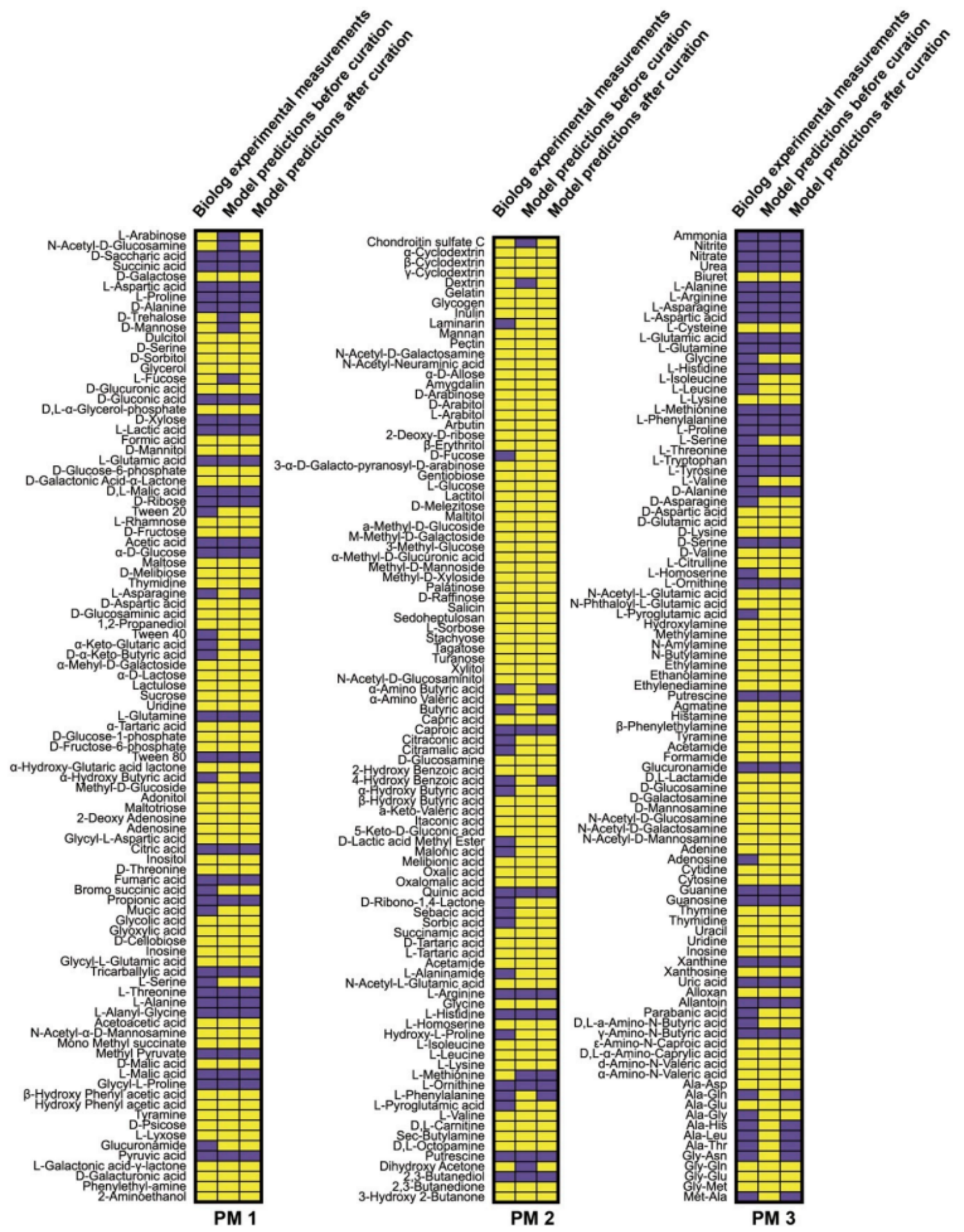

Figure 2. Comparison of Biolog experimental measurements (left columns) and model predictions before (middle columns) and after curation (right columns). Purple indicates valid growth (i.e., growth predicted by our model or based on the Biolog results) and yellow indicates no growth. Results from 190 carbon sources (PM1 and 2) and 95 nitrogen (PM3) sources are displayed. 


\subsection{Prediction of Essential Genes, Reactions, and Metabolites for Bacterial Growth}

In silico single gene deletion was conducted, followed by calculating the specific growth rate after iteratively removing each individual gene and its associated reactions. With the FBA approach, 102, 114, 134, and 134 genes were identified as essential for growth in LB, MH, M9C, and M9S, respectively; $95,110,126$, and 128 genes were predicted to be essential for the respective media with the MOMA approach. Overall, 99 and 94 core essential genes were discovered using FBA and MOMA, respectively, for the 4 growth conditions (Figure 3). These genes were involved in energy production, biosynthesis of cofactors and cell envelope, and metabolism of amino acids, nucleotides, and lipids. When the prediction of gene essentiality was compared with the AB5075 three-allele transposon mutant library, iAB5075 showed a high accuracy of $87.6 \%$ using FBA and 88.5\% with MOMA. Similarly, 155/143, 168/159, 200/189, and 200/191 reactions and 245/251, 255/259, 284/287, and 284/287 metabolites were considered essential for bacterial growth on LB, MH, M9C, and M9S media using FBA and MOMA, respectively (Figure 3 and Supplementary Materials Dataset 1). Comparison of FBA and MOMA core essential components revealed 89 core essential genes, 137 core essential reactions, and 237 core essential metabolites, indicating a substantially high agreement between both methods. Taken together, the prediction accuracies using draft $i \mathrm{AB} 5075$ obtained directly from CarveMe were only $72.8 \%$ and $74.3 \%$ for Biolog nutrient utilization and gene essentiality, respectively; whereas after curation, the corresponding prediction accuracies were significantly improved and achieved $86.3 \%$ and $87.6 \%$. Overall, $i$ AB5075 exhibits precise prediction of gene essentiality and thus can be utilized to systematically interrogate genotype-phenotype relationships.

(a).
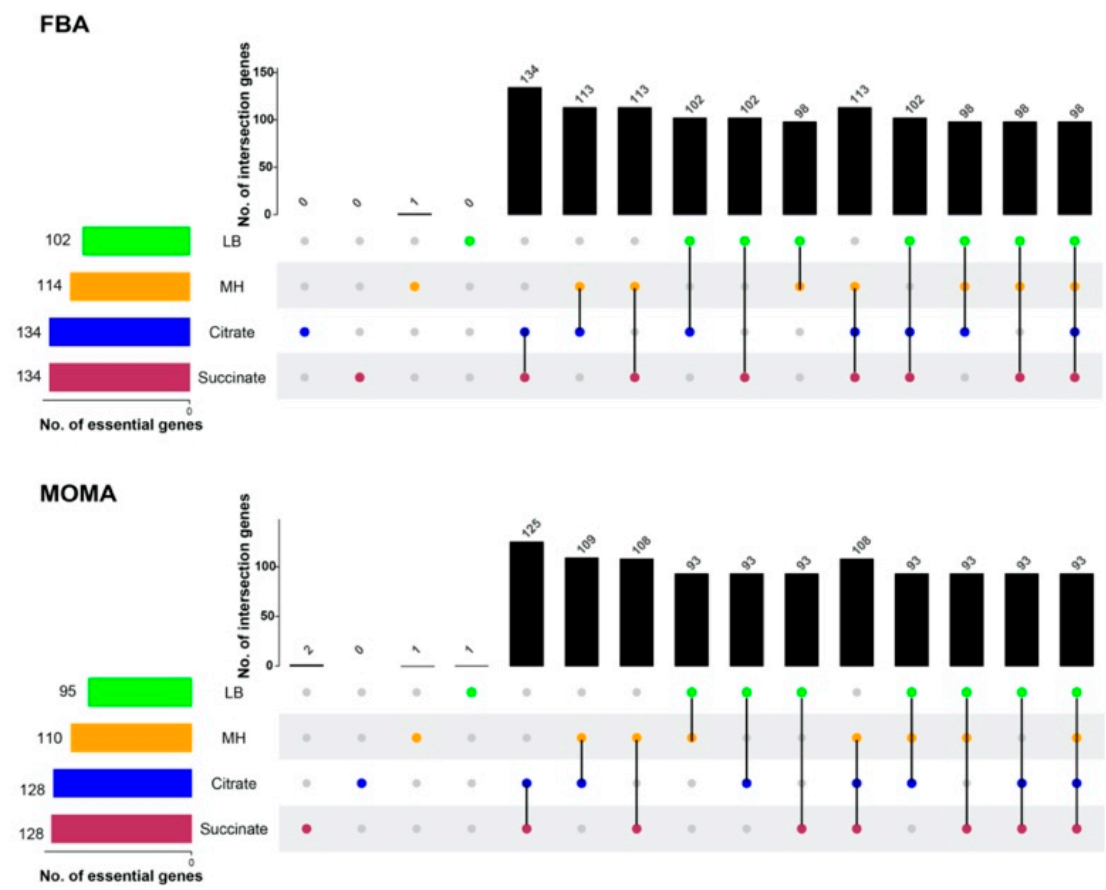

Figure 3. Cont. 
(b)

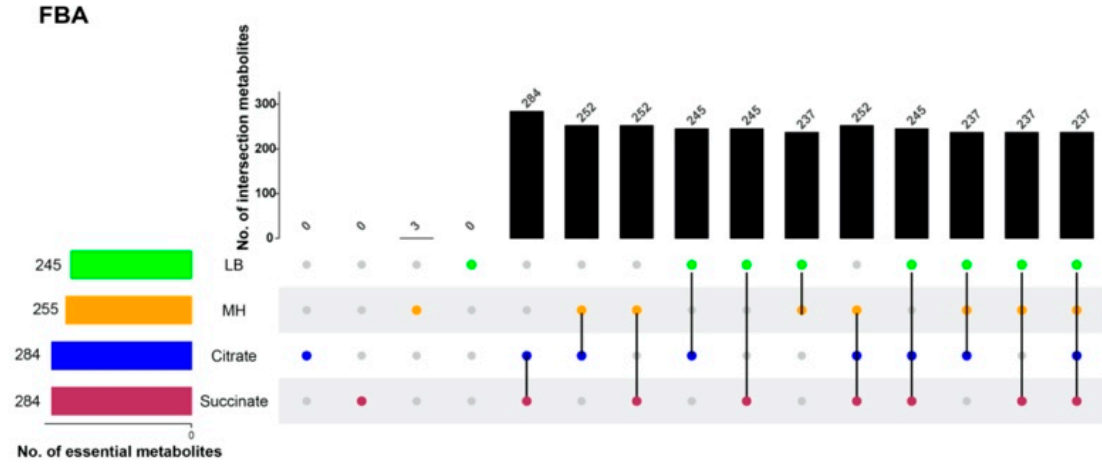

No. of essential metabolites

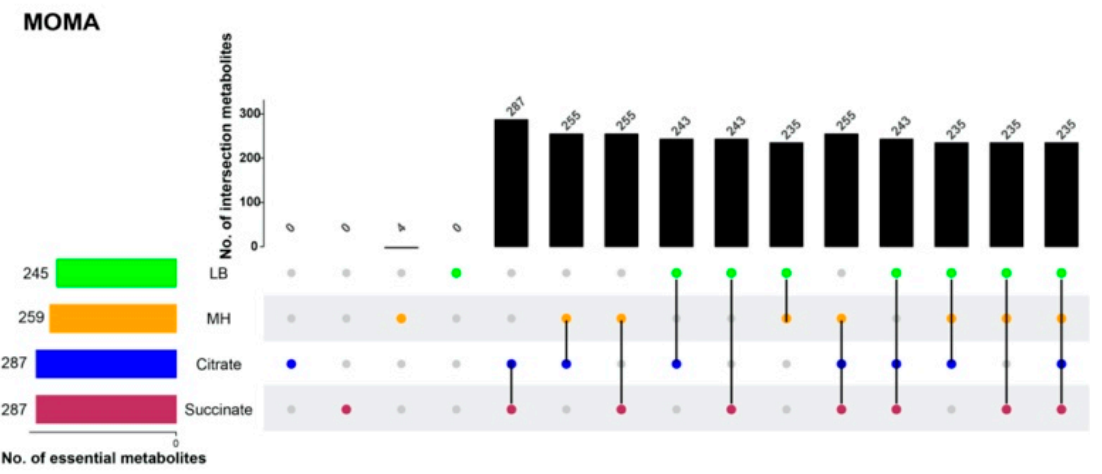

(c).
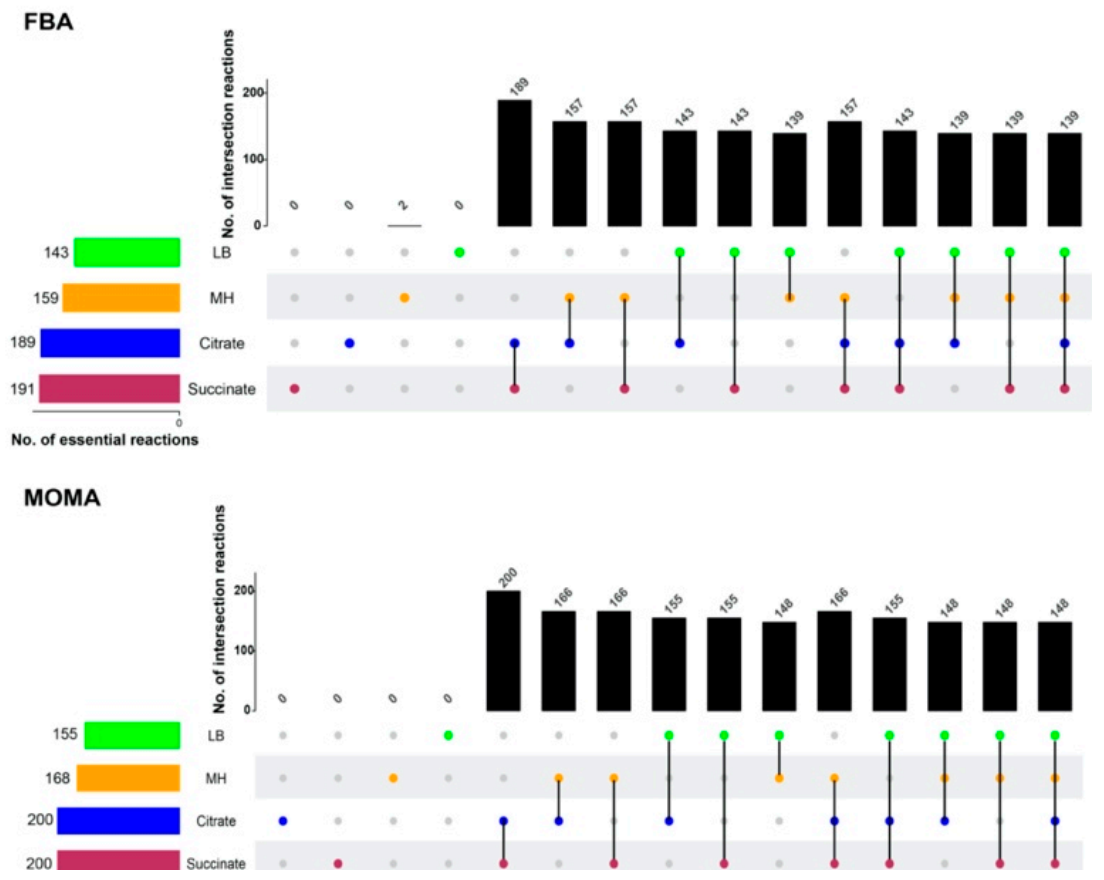

Figure 3. Essential genes, metabolites, and reactions predicted under four nutrient conditions using flux balance analysis (FBA) and minimization of metabolic adjustment (MOMA). The four nutrient conditions were LB media, MH media, M9 media supplemented with citrate (M9C), and M9 media supplemented with succinate (M9S). Results for FBA are shown at top and those for MOMA at bottom. (a) Essential genes, (b) essential metabolites, and (c) essential reactions. The circles represent different media (green, LB; orange, MH; blue, M9+citrate; red, M9+succinate). The vertical black bars represent the numbers of essential genes (a), metabolites (b), or reactions (c) uniquely or commonly identified for different media. The horizontal bars indicate the numbers of essential genes (a), metabolites (b), or reactions (c) totally identified for differential media. 


\subsection{Modeling Metabolic Changes of Strain AB5075 in a Murine Bacteremia Model with} Transcriptomic Constraints

A. baumannii may alter its metabolism to adapt to the host environment during infection [37]. However, our understanding of such complex metabolic responses is very limited. With a combination of fold change $>2$ and false discovery rate (FDR)-adjusted $p$ value $<0.05$, a total of 1408 genes in A. baumannii AB5075 were differentially expressed at $4 \mathrm{~h}$ post-infection compared to $2 \mathrm{~h}$, and 396 genes were mapped to iAB5075 (Table S1). Based on pathway enrichment analysis (Fisher's exact test, FDR-adjusted $p$-value $<0.05)$, the differentially expressed genes were mainly enriched in the following pathways: intracellular trafficking, secretion and vesicular transport; secondary metabolites biosynthesis, transport, and catabolism; inorganic ion transport and metabolism; and translation, ribosomal structure, and biogenesis. Among those genes mapped to $i \mathrm{AB} 5075,283$ were identified as being involved with transport and metabolism of carbohydrates, nucleotides, and amino acids; energy production and conversion; and cell envelope metabolism, indicating their critical roles in adaption to the host environment.

The transcriptomic data were then incorporated in the model as flux constraints to accurately predict metabolic fluxes using the INIT algorithm. In addition, constraints on bacterial growth rate and protein crowding were also imposed on the model. Specifically, 478 flux-carrying reactions were detected at $2 \mathrm{~h}$ post infection, while 502 reactions had non-zero fluxes at $4 \mathrm{~h}$ post infection. Compared to $2 \mathrm{~h}$ post infection, 198 significantly changed fluxes (fold change $>2$, FDR $<0.05$ ) were identified at $4 \mathrm{~h}$ post infection, including 146 increased and 52 decreased fluxes (Table S2). The increased fluxes were mainly detected in a broad range of metabolic pathways, including the tricarboxylic acid (TCA) cycle, gluconeogenesis, amino acid metabolism, and biosynthesis of peptidoglycan, lipopolysaccharide (LPS), nucleotides, and fatty acids.

In the TCA cycle, six of eight metabolic fluxes were significantly increased at $4 \mathrm{~h}$ post-infection compared to $2 \mathrm{~h}$ post-infection, while the flux through fumarate hydratase was significantly decreased (Figure 4a). Notably, the production flux through malate dehydrogenase was $1.11 \mathrm{mmol} \cdot \mathrm{gDW} \cdot \mathrm{h}^{-1}$ from malate to oxaloacetate $4 \mathrm{~h}$ post infection, whereas the flux from oxaloacetate to malate was $3.25 \mathrm{mmol} \cdot \mathrm{gDW} \cdot \mathrm{h}^{-1}$; the two metabolites (malate and oxaloacetate) were utilized by the gluconeogenesis pathway, resulting in significantly increased fluxes through gluconeogenesis (Figure $4 \mathrm{~b}$ ). At the same time, most fluxes through the pentose phosphate pathway (PPP) were significantly increased, whereas the flux from D-ribose 5-phospahte towards D-sedoheptulose 7-phosphate was significantly decreased (Figure 4b). Remarkably, the flux thorough 5-phospho- $\alpha$-D-ribose 1-diphosphate (PRPP) synthase was dramatically increased $\left(0 \mathrm{mmol} \cdot \mathrm{gDW} \cdot \mathrm{h}^{-1}\right.$ at $2 \mathrm{~h}$ post infection versus $0.24 \mathrm{mmol} \cdot \mathrm{gDW} \cdot \mathrm{h}^{-1}$ at $4 \mathrm{~h}$ post infection), and the increased PRPP was then utilized in the biosynthesis of purine and pyrimidine nucleotides. 


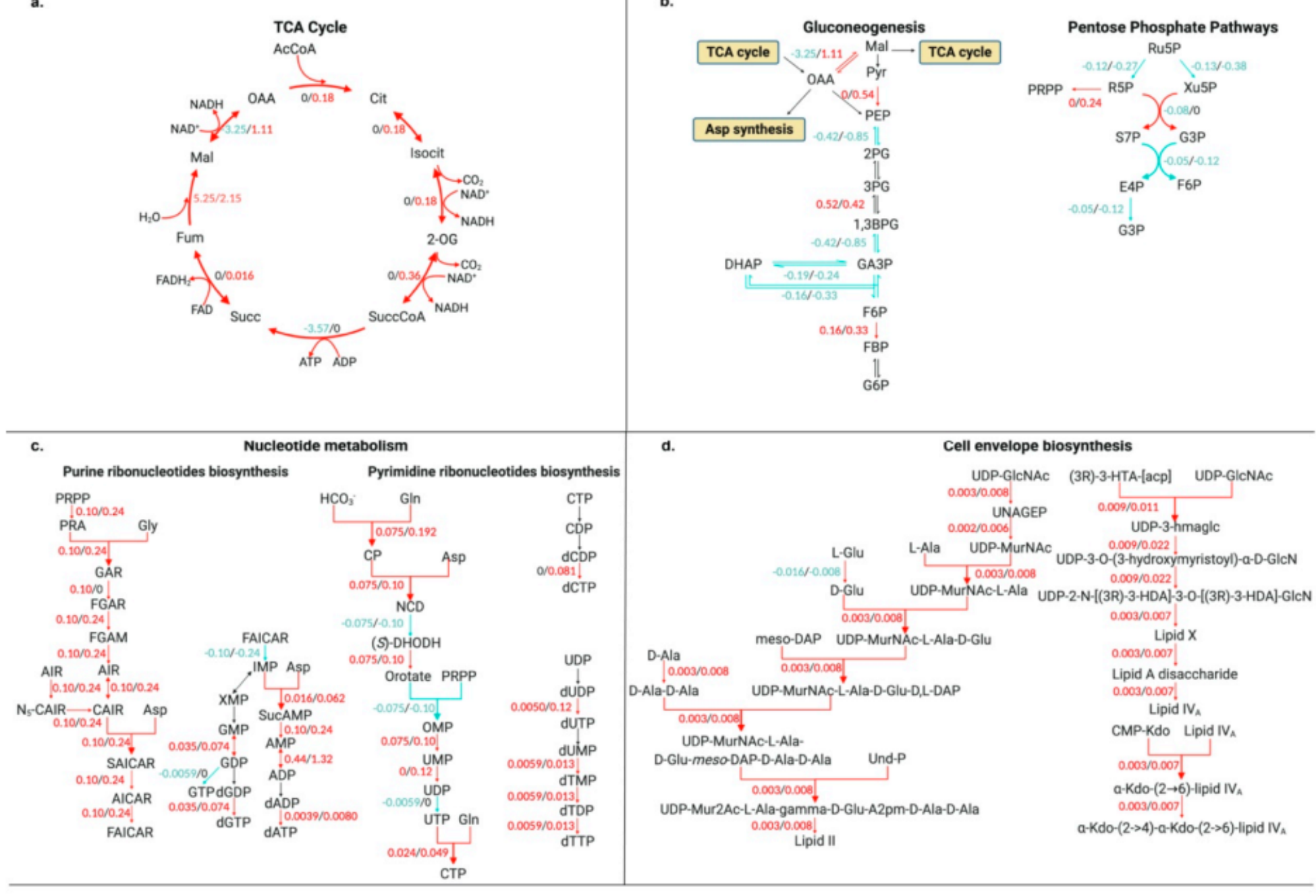


Figure 4. Differentially regulated metabolic fluxes in multiple pathways at $4 \mathrm{~h}$ post infection compared to $2 \mathrm{~h}$ post infection. Differentially regulated metabolic fluxes through (a) the TCA cycle, (b) the gluconeogenesis and pentose phosphate pathways, (c) nucleotide metabolism, and (d) cell structure biosynthesis. Significantly regulated fluxes (fold change $>2$ and FDR <0.05) are shown in color with red (produced fluxes) or blue (consumed fluxes). AcCoA, acetyl-CoA; CIT, citrate; Isocit, isocitrate; 2-OG, $\alpha$-ketoglutarate; SuccCoA, succinyl-CoA; Succ, succinate; Fum, fumarate; MAL, (S)-malate; OAA, oxaloacetate; Pyr, pyruvate; PEP, phosphoenolpyruvate; 2PG, 2-phosphoglycerate; 3PG, 3-phosphoglycerate; 1,3-BPG, 1,3-bisphosphoglycerate; GA3P, glyceraldehyde 3-phosphate; DHAP, dihydroxyacetone phosphate; F6P, fructose 6-phosphate; FBP, fructose 1,6-biphosphate; G6P, glucose 6-phosphate; R5P, ribose 5-phosphate; Ru5P, ribulose 5-phosphate; Xu5P, xylulose 5-phosphate; S7P, sedoheptulose 7-phosphate; PRPP, phosphoribosyl pyrophosphate; PRA, 5-phospho- $\beta$-D-ribosylamine; Gly, glycine; GAR, $N^{1}$-(5-phospho- $\beta$-D-ribosyl)glycinamide; FGAR, $N^{2}$-formyl- $N^{1}$-(5-phospho- $\beta$-D-ribosyl)glycinamide; FGAM, 2 -(formamido)- $N^{1}$-(5-phospho- $\beta$-D-ribosyl)acetamidine; AIR, 5-amino-1-(5-phospho- $\beta$-D-ribosyl)imidazole; CAIR, 5-amino-1-(5-phospho-D-ribosyl)imidazole-4-carboxylate; SAICAR, 5'-phosphoribosyl-4-(N-succinocarboxamide)5-aminoimidazole; AICAR, 5-amino-1-(5-phospho-D-ribosyl)imidazole-4-carboxamide; FAICAR, 5-formamido-1-(5-phospho-D-ribosyl)-imidazole-4-carboxamide; Asp, L-aspartate; SucAMP, adenylo-succinate; Gln, L-glutamine; CP, carbamoyl phosphate; NCD, N-carbamoyl-L-aspartate; (S)-DHODH, (S)-dihydroorotate; UDP-GlcNAc, UDP- $N$-acetyl- $\alpha$-D-glucosamine; UNAGEP, UDP- $N$-acetyl- $\alpha$-D-glucosamine-enolpyruvate; UDP-MurNAc, UDP- $N$-acetyl- $\alpha$-D-muramate; UDP-MurNAc-L-Ala, UDP$N$-acetyl- $\alpha$-D-muramoyl-L-alanine; UDP-MurNAc-L-Ala-D-Glu, UDP-N-acetyl- $\alpha$-D-muramoyl-L-alanyl-D-glutamate; meso-DAP, meso-diaminopimelate; UDP-MurNAcL-Ala-D-Glu-D,L-DAP, UDP-N-acetyl- $\alpha$-D-muramoyl-L-alanyl- $\gamma$-D-glutamyl-meso-2,6-diaminopimelate; UDP-MurNAc-L-Ala-D-Glu-meso-DAP-D-Ala-D-Ala, UDP-Nacetyl- $\alpha$-D-muramoyl-L-alanyl- $\gamma$-D-glutamyl-meso-2,6-diaminopimeloyl-D-alanyl-D-alanine; Und-P, di-trans,octa-cis-undecaprenyl phosphate; (3R)-3-HTA-[acp], (3R)-3hydroxytetradecanoyl-[acp]; UDP-3-hmaglc, UDP-3-O-[(3R)-3-hydroxydecanoyl]-N-acetyl- $\alpha$-D-glucosamine; UDP-3-O-(3-hydroxymyristoyl)- $\alpha$-D-GlcN, UDP-3-O(3-hydroxymyristoyl)- $N$-acetyl- $\alpha$-D-glucosamine; UDP-2- $N$-[(3R)-3-HDA]-3-O-[(3R)-3-HDA]-GlcN, UDP-2-N-[(3R)-3-hydroxydodecanoyl]-3-O-[(3R)-3-hydroxydecanoyl]$\alpha$-D-glucosamine. 
The increased fluxes in the PPP toward nucleotide metabolism were significantly increased $4 \mathrm{~h}$ post infection (Figure $4 \mathrm{~b}$ ). As the downstream utilization of PRPP, the overall fluxes via enzymes (e.g., amido phosphoribosyl transferase, phosphoribosyl amine-glycine ligase, and phosphoribosyl glycinamide formyltransferase) in inosine-5'-phosphate biosynthesis were increased, resulting in the increased production of dGTP and dATP from guanosine and adenosine ribonucleotide de novo biosynthesis (Figure 4c). Additionally, increased fluxes involving pyrimidine deoxyribonucleotide biosynthesis were also identified, which enhanced the production of dCTP and dTTP (Figure 4c).

At $4 \mathrm{~h}$ post-infection, there were remarkable increases in fluxes involving the biosynthesis of cell envelope components (e.g., peptidoglycan and LPS biosynthesis). At this time, most fluxes within peptidoglycan biosynthesis from UDP- $N$-acetyl- $\alpha$-D-glucosamine to meso-diaminopimelate containing lipid II were increased $266.6 \%$ to $300.0 \%$, whereas the flux over glutamate racemase was significantly decreased (Figure $4 \mathrm{~d}$ ). In lipid A biosynthesis, fluxes through acyl-ACP-UDP-N-acetylglucosamine $\mathrm{O}$-acyltransferase, UDP-3-O-acyl- $\mathrm{N}$-acetylglucosamine deacetylase, UDP-3-O-(3-hydroxymyristoyl) glucosamine $N$-acyltransferase, UDP-2,3-diacylglucosamine diphosphatase, lipid-A-disaccharide synthase, and tetraacyldisaccharide 4'-kinase were upregulated, resulting in a dramatic increase in the production of lipid A (Figure $4 \mathrm{~d}$ ).

In addition to the increased fluxes through the above metabolic pathways, fluxes through fatty acid biosynthesis and amino acid metabolism were also significantly impacted at both 2 and 4 h post infection. In fatty acid biosynthesis, most fluxes were significantly increased at $4 \mathrm{~h}$ compared to $2 \mathrm{~h}$ (Table S3a), whereas 71 key fluxes in amino acid metabolism were significantly affected at each time point (Table S3b).

\section{Discussion}

A. baumannii is a Gram-negative opportunistic pathogen which has imposed a heavy burden on the global health care system [38]. There is an urgent need to understand how A. baumannii responds to the host immune system during infection. GSMM has been increasingly employed to predict key genetic targets in order to guide therapeutic interventions and decipher the mechanism(s) of antibiotic killing and resistance. Unfortunately, few studies have previously examined bacterial metabolic responses in vivo using GSMMs, and none of these studies examined A. baumannii. We report here, for the first time, the development, validation, and application of a GSMM named iAB5075 for the MDR clinical isolate A. baumannii AB5075.

To the best of our knowledge, the only GSMMs previously developed for A. baumannii are for strains ATCC 19606 and AYE [14,16,39,40]. However, ATCC 19606 and AYE were isolated in the 1950s and 2003, respectively, and have significant differences in their genomic content and virulence phenotypes compared to more recent clinical isolates [41]. In addition, of both isolates, only AYE is MDR. Importantly, AB5075, the focus of the present study was a well-characterized modern clinical isolate that has been employed as a type strain of $A$. baumannii to investigate virulence and multidrug resistance [41-43]. Given this situation, we developed and validated an AB5075-specific GSMM, iAB5075 (Figures 2 and 3). Compared to the most recent GSMM in AYE (iCN718), iAB5075 contains a larger number of unique genes, metabolites, and reactions, and has a much higher prediction accuracy (i.e., $87.6 \%$ for $i \mathrm{AB} 5075$ compared to $80.2 \%$ for $i \mathrm{CN718)}$. These differences are mainly attributable to the different compartment settings, transport and exchange reactions contained within the two models (Table 1), and indicate that $i$ AB5075 represents the most comprehensive GSMM thus far developed for A. baumannii.

Transcriptomics is increasingly employed to examine the mechanisms underpinning bacterial pathogenesis and antibacterial resistance in Gram-negative pathogens in vitro and in vivo [13,44-46]. However, no study to date has integrated in vivo transcriptomic data with GSMM in A. baumannii. It is important to note that the nutrient complex composition varies from in vivo to in vitro based on nutrient conditions [47]. In the present study, the integration and simulation with in vivo transcriptomic data from the murine bloodstream infection model provide an important expansion of genes involved 
with adaption to the host environment. This, in turn, enabled a global view of metabolic responses to the host at the network level. Compared to $2 \mathrm{~h}$ post infection, $4 \mathrm{~h}$ post infection induced significant metabolic changes in biosynthesis of nucleotides, peptidoglycan, lipopolysaccharide, and fatty acids; and central carbon and amino acid metabolism.

When INIT algorithm was applied to the RNA-seq data, 198 fluxes were identified as critical for AB5075 to cause serious bloodstream infections in mice at $4 \mathrm{~h}$ post infection (Figure 4 ). These fluxes represent a novel set of metabolic functions, which are integral to the establishment of $A$. baumannii infections. Within central metabolism, the upregulated fluxes were associated with TCA cycle, gluconeogenesis, and pentose phosphate pathway (Figure $4 a, b)$. The increased production flux in TCA cycle via malate dehydrogenase at $4 \mathrm{~h}$ resulted in enhanced fluxes in gluconeogenesis (Figure $4 \mathrm{~b}$ ). The downstream metabolites from glucogenesis were subsequently utilized by PPP pathway, with most of the identified PPP fluxes being dramatically increased (Figure 4b). It has previously been shown that the TCA cycle and gluconeogenesis play key roles in the virulence of Salmonella enterica during infection [48]. A number of intermediates in the TCA cycle and gluconeogenesis have been identified to contribute to the virulence in macrophages in vivo [48]. Our observations suggest that the increased fluxes through central metabolic pathways might have important consequences for bloodstream infections, given that $A$. baumannii relies on these pathways to provide adequate energy metabolism and to contribute to the establishment and enhancement of infection.

A. baumannii can evade the host innate immune response using multiple virulence factors, such as surface glycoconjugates [49]. An important barrier that protects $A$. baumannii against host immune responses during infection is the outer membrane. In $A$. baumannii, the primary component of the outer leaflet of outer membrane is LPS, which consists of lipid A, a core oligosaccharide, and a polysaccharide $\mathrm{O}$-antigen. Model simulation showed that fluxes through lipid A biosynthesis were significantly increased (up to $267 \%$ ) $4 \mathrm{~h}$ post infection compared to $2 \mathrm{~h}$ post infection. In addition to lipid A biosynthesis, increased fluxes were also detected in peptidoglycan biosynthesis via the conversion of UDP- $N$-acetyl- $\alpha$-D-glucosamine to meso diaminopimelate containing lipid II. It has previously been shown that genes involved in the biosynthesis and maintenance of LPS and peptidoglycan contribute to the fitness of A. baumannii during bacteremia [9], and that both LPS and peptidoglycan contribute to bacterial cell stability and resistance to lysozyme in the host environment [50,51]. During infection, the significantly increased fluxes in LPS and peptidoglycan biosynthesis are in line with these previous findings, suggesting that both metabolic pathways contribute to bacterial fitness during infection in the murine host. The balance of LPS and fatty acid biosynthesis plays an important role in maintaining cell envelope function and integrity in A. baumannii [52]. Compared to $2 \mathrm{~h}$ post infection, most fluxes through fatty acids biosynthesis were significantly increased at $4 \mathrm{~h}$ post infection, consistent with the increased fluxes in LPS biosynthesis. These increased fluxes are crucial for rebalancing fatty acids and LPS to maintain membrane integrity.

In addition to surface glycoconjugates, other strategies also contribute to bacterial defense against host immune systems, including secreted proteins and multiple other regulators and metabolic pathways (e.g., OmpA porin, BfmR global regulator, phenylacetic acid catabolism pathways). Nucleotide second messengers (e.g., cAMP, cyclic di-GMP, and penta/tetra-guanosine phosphate) are key bacterial regulators involved in adaptations to conditions of limiting or non-optimal carbon and energy resources $[53,54]$. Cyclic di-GMP and other nucleotide second messengers control a variety of processes (e.g., production of exopolysaccharides, protein adhesins, pili and flagella, and cell differentiation) and have a central role in modulating virulence and persistence [55]. Increased fluxes were detected in inosine-5'-phosphate biosynthesis I, inosine-5' -phosphate biosynthesis II, and pyrimidine ribonucleotide biosynthesis (Figure 4c), indicating that AB5075 increased production of nucleotide to adapt to the host environment. As the downstream production in nucleotide metabolism, second messengers most likely contributed to the regulation of surface adaption and virulence factors that assisted with initiation of infections in the mice. Finally, fluxes via amino acid metabolism were 
significantly impacted in AB5075 during bacteremia. These effects might be attributed to the adaption of metabolic changes in multiple pathways.

Overall, fluxes were significantly changed through multiple pathways in AB5075 at $4 \mathrm{~h}$ post infection compared with $2 \mathrm{~h}$ post infection. Marked fluxes through central metabolism, nucleotide metabolism, and fatty acid and cell envelope biosynthesis might contribute to adaptations to the host environment and enhanced infection during bacteremia. These impacted pathways may be potential therapeutic targets for future drug development and therapy optimization.

\section{Conclusions}

In summary, we constructed and validated the first GSMM, iAB5075, for a modern model strain of MDR A. baumannii, namely AB5075. This is the first study to integrate in vivo transcriptomic data with GSMM, and importantly, the modeling provides a precise understanding of metabolic changes in A. baumannii in response to a host immune system during bacteremia. Model $i$ AB5075 provides a unique $>$ in silico platform for predicting bacterial metabolic responses to different treatments at the network level, which may assist in the optimization of antibiotic treatment in patients.

Supplementary Materials: The following are available online at http://www.mdpi.com/2076-2607/8/11/1793/s1.

Author Contributions: Conceptualization, J.Z.; Methodology, J.Z. and Y.Z.; Software, J.Z. and J.H.; Validation, J.Z., M.A. and F.S.; Formal Analysis, J.Z.; Investigation, J.Z. and Y.Z.; Resources, J.W. and K.C.; Data Curation, J.Z. and Y.-W.L.; Writing—Original Draft Preparation, J.Z.; Writing—Review and Editing, J.Z., Y.Z., T.V., F.S. and J.L.; Visualization, J.Z. and M.A.; Supervision, Y.Z. and J.L.; Project Administration, Y.Z. and J.L.; Funding Acquisition, J.L. All authors have read and agreed to the published version of the manuscript.

Funding: This study was supported by a research grant from the National Institute of Allergy and Infectious Diseases of the National Institutes of Health (R01 AI132681). J.L. is an Australian National Health and Medical Research Council (NHMRC) Principal Research Fellow and T.V. is an Australian NHMRC Industry Career Development Level 2 Research Fellow. The content is solely the responsibility of the authors and does not necessarily represent the official views of the National Institute of Allergy and Infectious Diseases or the National Institutes of Health.

Conflicts of Interest: The authors declare no conflict of interest.

\section{References}

1. Mulani, M.S.; Kamble, E.E.; Kumkar, S.N.; Tawre, M.S.; Pardesi, K.R. Emerging strategies to combat ESKAPE pathogens in the era of antimicrobial resistance: A review. Front. Microbiol. 2019, 10, 539. [CrossRef] [PubMed]

2. Dijkshoorn, L.; Nemec, A.; Seifert, H. An increasing threat in hospitals: Multidrug-resistant Acinetobacter baumannii. Nat. Rev. Microbiol. 2007, 5, 939-951. [CrossRef] [PubMed]

3. World Health Organization. WHO Publishes List of Bacteria for Which New Antibiotics Are Urgently Needed; WHO: Geneva, Switzerland, 2017.

4. Monserrat-Martinez, A.; Gambin, Y.; Sierecki, E. Thinking outside the bug: Molecular targets and strategies to overcome antibiotic resistance. Int. J. Mol. Sci. 2019, 20, 1255. [CrossRef] [PubMed]

5. Davies, J.; Davies, D. Origins and evolution of antibiotic resistance. Microbiol. Mol. Biol. Rev. 2010, 74, 417-433. [CrossRef]

6. Fang, F.C.; Frawley, E.R.; Tapscott, T.; Vázquez-Torres, A. Bacterial stress responses during host infection. Cell Host Microbe 2016, 20, 133-143. [CrossRef] [PubMed]

7. Totsika, M. Benefits and challenges of antivirulence antimicrobials at the dawn of the post-antibiotic era. Drug Deliv. Lett. 2016, 6, 30-37. [CrossRef]

8. Damron, F.H.; Oglesby-Sherrouse, A.G.; Wilks, A.; Barbier, M. Dual-seq transcriptomics reveals the battle for iron during Pseudomonas aeruginosa acute murine pneumonia. Sci. Rep. 2016, 6, 39172. [CrossRef]

9. Subashchandrabose, S.; Smith, S.; DeOrnellas, V.; Crepin, S.; Kole, M.; Zahdeh, C.; Mobley, H.L. Acinetobacter baumannii genes required for bacterial survival during bloodstream infection. mSphere 2016, 1, 00013-00015. [CrossRef]

10. Westermann, A.J.; Barquist, L.; Vogel, J. Resolving host-pathogen interactions by dual RNA-seq. PLoS Pathog. 2017, 13, e1006033. [CrossRef] 
11. Pisu, D.; Huang, L.; Grenier, J.K.; Russell, D.G. Dual RNA-Seq of Mtb-infected macrophages in vivo reveals ontologically distinct host-pathogen interactions. Cell Rep. 2020, 30, 335-350. [CrossRef]

12. Bhuiyan, M.S.; Ellett, F.; Murray, G.L.; Kostoulias, X.; Cerqueira, G.M.; Schulze, K.E.; Maifiah, M.H.M.; Li, J.; Creek, D.J.; Lieschke, G.J. Acinetobacter baumannii phenylacetic acid metabolism influences infection outcome through a direct effect on neutrophil chemotaxis. Proc. Natl. Acad. Sci. USA 2016, 113, 9599-9604. [CrossRef] [PubMed]

13. Murray, G.L.; Tsyganov, K.; Kostoulias, X.P.; Bulach, D.M.; Powell, D.; Creek, D.J.; Boyce, J.D.; Paulsen, I.T.; Peleg, A.Y. Global gene expression profile of Acinetobacter baumannii during bacteremia. J. Infect. Dis. 2017, 215, S52-S57. [CrossRef] [PubMed]

14. Zhu, Y.; Lu, J.; Zhao, J.; Zhang, X.; Heidi, H.Y.; Velkov, T.; Li, J. Complete genome sequence and genome-scale metabolic modelling of Acinetobacter baumannii type strain ATCC 19606. Int. J. Med. Microbiol. 2020, 151412. [CrossRef] [PubMed]

15. Zhu, Y.; Zhao, J.; Maifiah, M.H.M.; Velkov, T.; Schreiber, F.; Li, J. Metabolic responses to polymyxin treatment in Acinetobacter baumannii ATCC 19606: Integrating transcriptomics and metabolomics with genome-scale metabolic modeling. mSystems 2019, 4, e00157-18. [CrossRef] [PubMed]

16. Presta, L.; Bosi, E.; Mansouri, L.; Dijkshoorn, L.; Fani, R.; Fondi, M. Constraint-based modeling identifies new putative targets to fight colistin-resistant A. baumannii infections. Sci. Rep. 2017, 7, 1-12. [CrossRef]

17. Hadadi, N.; Pandey, V.; Chiappino-Pepe, A.; Morales, M.; Gallart-Ayala, H.; Mehl, F.; Ivanisevic, J.; Sentchilo, V.; van der Meer, J.R. Mechanistic insights into bacterial metabolic reprogramming from omics-integrated genome-scale models. NPJ Syst. Biol. Appl. 2020, 6, 1-11. [CrossRef] [PubMed]

18. National Health and Medical Research Council (NHMRC). Australian Code of Practice for the Care and Use of Animals for Scientific Purposes; National Health and Medical Research Council: Canberra, Australia, 2004.

19. Zhao, J.; Li, M.; Wang, J.; Velkov, T.; Li, J. Dual RNA-seq revealed complex host-pathogen-polymyxin interactions in Acinetobacter baumannii bacteraemia. In Proceedings of the 29th European Congress of Clinical Microbiology \& Infectious Diseases, Amsterdam, The Netherlands, 13 April 2019.

20. Brittnacher, M.J.; Fong, C.; Hayden, H.; Jacobs, M.; Radey, M.; Rohmer, L. PGAT: A multistrain analysis resource for microbial genomes. Bioinformatics 2011, 27, 2429-2430. [CrossRef]

21. Machado, D.; Andrejev, S.; Tramontano, M.; Patil, K.R. Fast automated reconstruction of genome-scale metabolic models for microbial species and communities. Nucleic Acids Res. 2018, 46, 7542-7553. [CrossRef]

22. Hucka, M.; Finney, A.; Sauro, H.M.; Bolouri, H.; Doyle, J.C.; Kitano, H.; Arkin, A.P.; Bornstein, B.J.; Bray, D.; Cornish-Bowden, A. The systems biology markup language (SBML): A medium for representation and exchange of biochemical network models. Bioinformatics 2003, 19, 524-531. [CrossRef]

23. Heirendt, L.; Arreckx, S.; Pfau, T.; Mendoza, S.N.; Richelle, A.; Heinken, A.; Haraldsdóttir, H.S.; Wachowiak, J.; Keating, S.M.; Vlasov, V. Creation and analysis of biochemical constraint-based models using the COBRA Toolbox v. 3.0. Nat. Protoc. 2019, 14, 639-702. [CrossRef]

24. Zhu, Y.; Czauderna, T.; Zhao, J.; Klapperstueck, M.; Maifiah, M.H.M.; Han, M.-L.; Lu, J.; Sommer, B.; Velkov, T.; Lithgow, T. Genome-scale metabolic modeling of responses to polymyxins in Pseudomonas aeruginosa. GigaScience 2018, 7, giy021. [CrossRef] [PubMed]

25. Matthews, B.W. Comparison of the predicted and observed secondary structure of T4 phage lysozyme. Biochim. Biophys. Acta 1975, 405, 442-451. [CrossRef]

26. Gallagher, L.A.; Ramage, E.; Weiss, E.J.; Radey, M.; Hayden, H.S.; Held, K.G.; Huse, H.K.; Zurawski, D.V.; Brittnacher, M.J.; Manoil, C. Resources for genetic and genomic analysis of emerging pathogen Acinetobacter baumannii. J. Bacteriol. 2015, 197, 2027-2035. [CrossRef]

27. Agren, R.; Bordel, S.; Mardinoglu, A.; Pornputtapong, N.; Nookaew, I.; Nielsen, J. Reconstruction of genome-scale active metabolic networks for 69 human cell types and 16 cancer types using INIT. PLoS Comput. Biol. 2012, 8, e1002518. [CrossRef]

28. Robinson, M.D.; McCarthy, D.J.; Smyth, G.K. edgeR: A Bioconductor package for differential expression analysis of digital gene expression data. Bioinformatics 2010, 26, 139-140. [CrossRef]

29. Jeske, L.; Placzek, S.; Schomburg, I.; Chang, A.; Schomburg, D. BRENDA in 2019: A European ELIXIR core data resource. Nucleic Acids Res. 2019, 47, D542-D549. [CrossRef]

30. Wang, C.-H.; Zhang, C.; Xing, X.-H. Xanthine dehydrogenase: An old enzyme with new knowledge and prospects. Bioengineered 2016, 7, 395-405. [CrossRef] 
31. Yuan, H.; Chai, S.C.; Lam, C.K.; Xu, H.H.; Ye, Q.-Z. Two methionine aminopeptidases from Acinetobacter baumannii are functional enzymes. Bioorg. Med. Chem. Lett. 2011, 21, 3395-3398. [CrossRef]

32. Huynh, K.-H.; Hong, M.-k.; Lee, C.; Tran, H.-T.; Lee, S.H.; Ahn, Y.-J.; Cha, S.-S.; Kang, L.-W. The crystal structure of the D-alanine-D-alanine ligase from Acinetobacter baumannii suggests a flexible conformational change in the central domain before nucleotide binding. J. Microbiol. 2015, 53, 776-782. [CrossRef]

33. Sorci, L.; Blaby, I.; De Ingeniis, J.; Gerdes, S.; Raffaelli, N.; de Crécy Lagard, V.; Osterman, A. Genomics-driven reconstruction of Acinetobacter NAD metabolism insights for antibacterial target selection. J. Biol. Chem. 2010, 285, 39490-39499. [CrossRef]

34. Mo, M.L.; Palsson, B.Ø.; Herrgård, M.J. Connecting extracellular metabolomic measurements to intracellular flux states in yeast. BMC Syst. Biol. 2009, 3, 37. [CrossRef]

35. Saa, P.A.; Nielsen, L.K. 1l-ACHRB: A scalable algorithm for sampling the feasible solution space of metabolic networks. Bioinformatics 2016, 32, 2330-2337. [CrossRef]

36. Runci, F.; Gentile, V.; Frangipani, E.; Rampioni, G.; Leoni, L.; Lucidi, M.; Visaggio, D.; Harris, G.; Chen, W.; Stahl, J. Contribution of active iron uptake to Acinetobacter baumannii pathogenicity. Infect. Immun. 2019, 87, e00755-18. [CrossRef]

37. Mortensen, B.L.; Skaar, E.P. Host-microbe interactions that shape the pathogenesis of A cinetobacter baumannii infection. Cell. Microbiol. 2012, 14, 1336-1344. [CrossRef]

38. Peleg, A.Y.; Seifert, H.; Paterson, D.L. Acinetobacter baumannii: Emergence of a successful pathogen. Clin. Microbiol. Rev. 2008, 21, 538-582. [CrossRef]

39. Norsigian, C.J.; Kavvas, E.; Seif, Y.; Palsson, B.O.; Monk, J.M. iCN718, an updated and improved genome-scale metabolic network reconstruction of Acinetobacter baumannii AYE. Front. Genet. 2018, 9, 121. [CrossRef]

40. Rahim, N.A.; Cheah, S.-E.; Johnson, M.D.; Yu, H.; Sidjabat, H.E.; Boyce, J.; Butler, M.S.; Cooper, M.A.; Fu, J.; Paterson, D.L.; et al. Synergistic killing of NDM-producing MDR Klebsiella pneumoniae by two 'old' antibiotics-polymyxin B and chloramphenicol. J. Antimicrob. Chemother. 2015, 70, 2589-2597. [CrossRef]

41. Jacobs, A.C.; Thompson, M.G.; Black, C.C.; Kessler, J.L.; Clark, L.P.; McQueary, C.N.; Gancz, H.Y.; Corey, B.W.; Moon, J.K.; Si, Y. AB5075, a highly virulent isolate of Acinetobacter baumannii, as a model strain for the evaluation of pathogenesis and antimicrobial treatments. mBio 2014, 5, e01076-14. [CrossRef]

42. Wu, X.; Chavez, J.D.; Schweppe, D.K.; Zheng, C.; Weisbrod, C.R.; Eng, J.K.; Murali, A.; Lee, S.A.; Ramage, E.; Gallagher, L.A. In vivo protein interaction network analysis reveals porin-localized antibiotic inactivation in Acinetobacter baumannii strain AB5075. Nat. Commun. 2016, 7, 1-14. [CrossRef]

43. Tipton, K.A.; Rather, P.N. An ompR-envZ two-component system ortholog regulates phase variation, osmotic tolerance, motility, and virulence in Acinetobacter baumannii strain AB5075. J. Bacteriol. 2017, 199, e00705-16. [CrossRef]

44. Khaledi, A.; Schniederjans, M.; Pohl, S.; Rainer, R.; Bodenhofer, U.; Xia, B.; Klawonn, F.; Bruchmann, S.; Preusse, M.; Eckweiler, D. Transcriptome profiling of antimicrobial resistance in Pseudomonas aeruginosa. Antimicrob. Agents Chemother. 2016, 60, 4722-4733. [CrossRef]

45. Chan, J.P.; Wright, J.R.; Wong, H.T.; Ardasheva, A.; Brumbaugh, J.; McLimans, C.; Lamendella, R. Using bacterial transcriptomics to investigate Targets of Host-Bacterial Interactions in Caenorhabditis elegans. Sci. Rep. 2019, 9, 1-12. [CrossRef]

46. Qin, H.; Lo, N.W.-S.; Loo, J.F.-C.; Lin, X.; Yim, A.K.-Y.; Tsui, S.K.-W.; Lau, T.C.-K.; Ip, M.; Chan, T.-F. Comparative transcriptomics of multidrug-resistant Acinetobacter baumannii in response to antibiotic treatments. Sci. Rep. 2018, 8, 3515. [CrossRef]

47. Son, M.S.; Matthews, W.J.; Kang, Y.; Nguyen, D.T.; Hoang, T.T. In vivo evidence of Pseudomonas aeruginosa nutrient acquisition and pathogenesis in the lungs of cystic fibrosis patients. Infect. Immun. 2007, 75, 5313-5324. [CrossRef]

48. Yimga, M.T.; Leatham, M.P.; Allen, J.H.; Laux, D.C.; Conway, T.; Cohen, P.S. Role of gluconeogenesis and the tricarboxylic acid cycle in the virulence of Salmonella enterica serovar Typhimurium in BALB/c mice. Infect. Immun. 2006, 74, 1130-1140. [CrossRef]

49. Harding, C.M.; Hennon, S.W.; Feldman, M.F. Uncovering the mechanisms of Acinetobacter baumannii virulence. Nat. Rev. Microbiol. 2018, 16, 91. [CrossRef]

50. Auer, G.K.; Weibel, D.B. Bacterial cell mechanics. Biochemistry 2017, 56, 3710-3724. [CrossRef] 
51. Sukhithasri, V.; Nisha, N.; Biswas, L.; Kumar, V.A.; Biswas, R. Innate immune recognition of microbial cell wall components and microbial strategies to evade such recognitions. Microbiol. Res. 2013, 168, 396-406. [CrossRef]

52. Bojkovic, J.; Richie, D.L.; Six, D.A.; Rath, C.M.; Sawyer, W.S.; Hu, Q.; Dean, C.R. Characterization of an Acinetobacter baumannii lptD deletion strain: Permeability defects and response to inhibition of lipopolysaccharide and fatty acid biosynthesis. J. Bacteriol. 2016, 198, 731-741. [CrossRef]

53. Hengge, R. Linking bacterial growth, survival, and multicellularity-small signaling molecules as triggers and drivers. Curr. Opin. Microbiol. 2020, 55, 57-66. [CrossRef]

54. Whiteley, A.T.; Eaglesham, J.B.; de Oliveira Mann, C.C.; Morehouse, B.R.; Lowey, B.; Nieminen, E.A.; Danilchanka, O.; King, D.S.; Lee, A.S.; Mekalanos, J.J. Bacterial cGAS-like enzymes synthesize diverse nucleotide signals. Nature 2019, 567, 194-199. [CrossRef]

55. Hengge, R.; Gründling, A.; Jenal, U.; Ryan, R.; Yildiz, F. Bacterial signal transduction by cyclic di-GMP and other nucleotide second messengers. J. Bacteriol. 2016, 198, 15-26. [CrossRef]

Publisher's Note: MDPI stays neutral with regard to jurisdictional claims in published maps and institutional affiliations.

(C) 2020 by the authors. Licensee MDPI, Basel, Switzerland. This article is an open access article distributed under the terms and conditions of the Creative Commons Attribution (CC BY) license (http://creativecommons.org/licenses/by/4.0/). 